\title{
Adenosine deaminase (ADA) isoenzyme analysis in pleural effusions: diagnostic role, and relevance to the origin of increased ADA in tuberculous pleurisy
}

\author{
L. Valdés*, E. San José**, D. Alvarez*, J.M. Valle*
}

\begin{abstract}
Adenosine deaminase (ADA) isoenzyme analysis in pleural effusions: diagnostic role, and relevance to the origin of increased ADA in tuberculous pleurisy. L. Valdés, E. San José, D. Alvarez, J.M. Valle. CERS Journals Ltd 1996.

ABSTRACT: The rise in adenosine deaminase (ADA) activity in the pleural fluid of tuberculous pleurisy patients, though used for diagnosis, is of unknown origin. In this work, we determined ADA activity and the activities of 2'-deoxyadenosine deaminase and ADA-2 in 350 patients. We also considered whether the results throw light on the origin of high pleural fluid ADA in tuberculous pleurisy and estimated the diagnostic efficiency of 2'-deoxyadenosine deaminase, ADA-2 and total ADA activities with and without the inclusion of the 2'-deoxyadenosine deaminase/ADA activity ratio in a combined criterion.

The 350 pleural effusions were classified by previously established criteria as transudates (60 males/18 females) or as tuberculous (49 males/27 females), neoplastic (50 males/39 females), parapneumonic (36 males/19 females), empyematous (11 males/ 3 females), or miscellaneous ( 25 males/13 females) exudates. Total ADA, ADA-2 and 2 '-deoxyadenosine deaminase activities were, respectively, 127.5 $\pm 2.9,103 \pm 29.5$ and $42.8 \pm 14 \mathrm{U} \cdot \mathrm{L}^{-1}$ in tuberculous exudates. With diagnostic thresholds of 47,40 and 22 $U \cdot L^{-1}$ respectively, the sensitivities of $A D A, A D A-2$ and 2 -deoxyadenosine deaminase for tuberculosis were 100, 100 and 95\%; their specificities 91, 96 and 92\%; and their efficiencies 93, 97 and $93 \%$, respectively. One hundred and one effusions (all 76 tuberculous, 12 neoplastic, 4 parapneumonic and 9 empyematous exudates) had total ADA levels $>47 \mathrm{U} \cdot \mathrm{L}^{-1}$; of these, 8 neoplastic, 1 parapneumonic and all the tuberculous exudates had a 2 '-deoxyadenosine deaminase/ADA activity ratio $<0.49$. The criterion of simultaneously having ADA $>47 \mathrm{U} \cdot \mathrm{L}^{-1}, \mathrm{ADA}-2>40 \mathrm{U} \cdot \mathrm{L}^{-1}$ and a $2^{\prime}-$ deoxyadenosine deaminase/ADA activity ratio $<0.49$ was satisfied by all the tuberculous effusions but only eight others (all neoplastic) (sensitivity 100\%, specificity $97 \%$, efficiency $98 \%$ ).

We conclude that: 1) high total ADA activity in tuberculous pleural effusions is due mainly to an increase in ADA-2, and, therefore, originated from the only known source monocytes and macrophages; 2) ADA-2 was a more efficient diagnostic marker of tuberculous pleurisy than total ADA activity, although the difference was not statistically significant; and 3) among effusions with high total ADA the 2'deoxyadenosine deaminase/ADA activity ratio differentiates tuberculous effusions from empyemas and parapneumonic effusions, but fails to discriminate well between tuberculous and neoplastic effusions.
\end{abstract}

Eur Respir J., 1996, 9, 747-751.

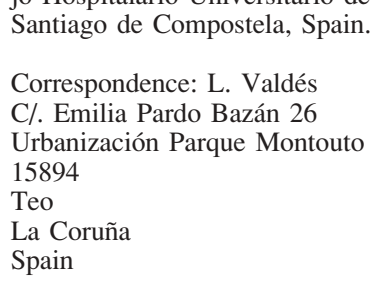
Santiago de Compostela, Spain.

Correspondence: L. Valdés

C/. Emilia Pardo Bazán 26

Urbanización Parque Montouto 15894

Teo

La Coruña

Spain

*Sección de Neumología and **Servicio de Bioquímica, Hospital de Conxo, Complejo Hospitalario Universitario de Santiago,

Keywords: Adenosine deaminase adenosine deaminase isoenzymes pleural effusion

tuberculous pleurisy

Received: December 201994

Accepted after revision December 101995

Presented in part at the European Respiratory Society Annual Congress, Nice, France, October 1994.
Adenosine deaminase (ADA) catalyses the conversion of adenosine to inosine, a stage of purine metabolism. Since 1978, when ADA activity was found to be high in tuberculous pleural exudates [1], ADA has been used in the diagnosis of tuberculous pleural effusions [2-5]; overall, its sensitivity in this role has been $99 \%$ and its specificity $93 \%$ [6]. High ADA levels can also be found in pleural effusions secondary to other processes or lesions, especially pneumonia, empyema, lymphoma, neoplasia and systemic lupus erythematosus [6].

ADA has two principal isoenzymes, ADA-1 and ADA2 , which have different optimal $\mathrm{pH}$, Michaelis constants and relative substrate specificity patterns [7]. ADA-1 has roughly equal affinities for adenosine and 2'-deoxyadenosine, with a 2'-deoxyadenosine deaminase/ ADA activity ratio of approximately 0.75 ; it is found in many tissues. ADA-2 has much greater affinity for adenosine (2'- deoxyadenosine deaminase/ADA activity ratio approximately 0.25 ), and is found only in macrophages [8], which release it when stimulated by the presence of live micro-organisms in their interior [9]. The high ADA activities in tuberculous pleural effusions are largely due to ADA-2, in view of which GAKIS and co-workers [10] have proposed that a low 2'-deoxyadenosine deaminase/ADA activity ratio be used as a criterion for distinguishing between tuberculous pleural 
effusions and other effusions with high total ADA activity.

In this work, we determined ADA activity and the activities of 2'-deoxyadenosine deaminase and ADA-2 in 350 pleural effusions; considered whether the results threw light on the origin of high ADA activity in tuberculous pleurisy; and estimated the diagnostic efficiencies of 2'-deoxyadenosine deaminase, ADA-2 and total ADA activity with and without the inclusion of the 2'-deoxyadenosine deaminase/ADA activity ratio in a combined criterion.

\section{Materials and methods}

We studied 350 consecutive cases of patients admitted to our centre on account of a pleural effusion for which a definitive diagnosis was ultimately reached. The diagnostic criteria used were as follows. Congestive heart failure: compatible clinical and radiological findings, remission upon appropriate treatment, and absence of pulmonary infiltrates, chest pain, thrombophlebitis and purulent sputum. Tuberculosis: presence of acid-fast bacilli in pleural fluid or biopsy tissue, or presence of necrotic caseous granulomas in biopsy tissue. Neoplasia: neoplastic tissue found in the pleural cavity by means of biopsy and/or cytology. Parapneumonic origin: association with pneumonia, lung abscess or bronchiectasis. Empyema: presence of purulent fluid, or positive culture of parapneumonic effusion. Miscellaneous exudates: pulmonary thromboembolism, surgery, systemic lupus erythematosus, pancreatitis, pleuropericarditis, hypothyroidism, yellow nail syndrome, chylothorax, haemothorax, Meigs' syndrome and benign asbestosis were also identified as causes of pleural effusions following pre-established criteria [11]. On the basis of these diagnoses, six groups were distinguished: Group I, transudates $(\mathrm{n}=78,60$ males $/ 18$ females, aged $71 \pm 11$ yrs); Group II, tuberculous exudates $(n=76$, 49 males/27 females, aged $34 \pm 18$ yrs); Group III, neoplastic exudates $(n=89,50$ males $/ 39$ females, aged $65 \pm 14$ yrs); Group IV, parapneumonic exudates ( $\mathrm{n}=55,36$ males/ 19 females, aged $66 \pm 18$ yrs); Group V, empyemas $(n=14$, 11 males/3 females, aged $52 \pm 18$ yrs); and Group VI, miscellaneous exudates $(n=38,25$ males $/ 13$ females, aged $54 \pm 19 \mathrm{yrs}$ ); (table 1). All patients were immunocompetent.

Pleural fluid and blood samples were taken at the same time from fasting patients. Both samples were centrifuged for $10 \mathrm{~min}$ at $1,000 \times \mathrm{g}$, and the supernatants were stored at $-40^{\circ} \mathrm{C}$ pending assay. Pleural biopsies were performed with a COPE needle [12] or ABRAMs needle [13], except when a transudate was suspected.

ADA and 2'-deoxyadenosine deaminase activities were determined colorimetrically as per GIUSTI [14], ADA isoenzymes being distinguished by their different affinities for adenosine and 2'-deoxyadenosine. Briefly, duplicate $25 \mu \mathrm{L}$ samples were incubated for $60 \mathrm{~min}$ at $37^{\circ} \mathrm{C}$ with $500 \mu \mathrm{L}$ of $21 \mathrm{mM}$ adenosine (one sample) or $21 \mathrm{mM} 2^{\prime}-$ deoxyadenosine (the other) in $50 \mathrm{mM}$ phosphate buffer [15], and the ammonium ion released was determined by reaction for $30 \mathrm{~min}$ with $1.5 \mathrm{~mL}$ of phenol nitroprusside $(106 \mathrm{mM}$ phenol plus $0.17 \mathrm{mM}$ sodium nitroprusside) in the presence of $1.5 \mathrm{~mL}$ of sodium hypochlorite (11 $\mathrm{mM} \mathrm{NaOCl}$ plus $125 \mathrm{mM} \mathrm{NaOH}$ ), absorption at 628
Table 1. - Aetiology of pleural effusions

\begin{tabular}{|c|c|c|c|}
\hline Aetiology & $\begin{array}{c}\text { Sex } \\
\text { M:F ratio }\end{array}$ & $\begin{array}{l}\text { Age* } \\
\text { yrs }\end{array}$ & $\mathrm{n}$ \\
\hline Transudates & 3.3 & $71 \pm 11$ & 78 \\
\hline Heart failure & & & 61 \\
\hline Liver cirrhosis & & & 10 \\
\hline Superior vena cava syndrome & & & 3 \\
\hline Hypoalbuminaemia & & & 3 \\
\hline Massive atelectasis & & & 1 \\
\hline Tuberculosis & 1.8 & $34 \pm 18$ & 76 \\
\hline Neoplastic & 1.3 & $65 \pm 14$ & 89 \\
\hline Metastasis & & & 55 \\
\hline Lymphoma & & & 16 \\
\hline Chronic lymphatic leukaemia & & & 3 \\
\hline Mesothelioma & & & 2 \\
\hline Undetermined & & & 13 \\
\hline Parapneumonics & 1.9 & $66 \pm 18$ & 55 \\
\hline Empyema & 3.6 & $52 \pm 18$ & 14 \\
\hline Miscellaneous & 1.9 & $54 \pm 19$ & 38 \\
\hline Pulmonary thromboembolism & & & 11 \\
\hline Postsurgery & & & 10 \\
\hline Systemic lupus erythematosus & & & 5 \\
\hline Pancreatitis & & & 4 \\
\hline Pleuropericarditis & & & 2 \\
\hline Hypothyroidism & & & 1 \\
\hline Yellow nail syndrome & & & 1 \\
\hline Cylothorax & & & 1 \\
\hline Haemothorax & & & 1 \\
\hline Meigs' syndrome & & & 1 \\
\hline Benign asbestosis & & & 1 \\
\hline
\end{tabular}

*: mean \pm SD

$\mathrm{nm}$ then being read in a spectrophotometer. To control for ammonium present before addition of exogenous adenosine, untreated samples were run in parallel. Estimated ADA-2 activity was calculated from the ADA and 2'-deoxyadenosine deaminase activities using the affinity of ADA-2 for the two substrates [15]. The results were expressed in $\mathrm{U} \cdot \mathrm{L}^{-1}$.

\section{Statistical analysis}

Distributions were deemed normal or non-normal on the basis of their skew and kurtosis. The statistical significance of differences between means was estimated using Student's t-test for normal distributions and Wilcoxon's rank sum test otherwise. The diagnostic value of the parameters studied was assessed in terms of sensitivity, specificity and efficiency. The diagnostic thresholds for each diagnostic parameter considered were defined as the values affording greatest diagnostic efficiency.

\section{Results}

Table 2 lists the means and standard deviations of total ADA, ADA-2 and 2'-deoxyadenosine deaminase activities in pleural fluid for each group of patients studied. The intermean differences between the tuberculous group and each of the others are statistically significant for all three parameters $(p<0.05$ for 2'-deoxyadenosine deaminase with respect to the neoplastic group; $\mathrm{p}<0.001$ 
Table 2. - Total adenosine deaminase, adenosine deaminase-2 and 2'-deoxyadenosine deaminase activities in pleural fluid

\begin{tabular}{lccc}
\hline Aetiology & ADA & ADA-2 & $\begin{array}{c}\text { 2'-deoxyadenosine } \\
\text { deaminase } \\
\text { U.L-1 }\end{array}$ \\
& U.L-1 & U.L-1 & $4.3 \pm 2.5^{*}$ \\
Transudates & $12.1 \pm 7.2^{*}$ & $9.3 \pm 6.7^{*}$ & $42.8 \pm 14$ \\
Tuberculosis & $127.5 \pm 32.9$ & $103.5 \pm 29.5$ & $21.7 \pm 82.7^{* *}$ \\
Neoplastics & $42.1 \pm 107.5^{*}$ & $20.4 \pm 13.8^{*}$ & $21.5 \pm 7.9^{*}$ \\
Parapneumonics & $27.4 \pm 12.2^{*}$ & $18.5 \pm 8^{*}$ & $11.5 \pm 120.3$ \\
Empyemas & $135.3 \pm 166.8$ & $25.8 \pm 19.5^{*}$ & $88.9 \pm 120.3$ \\
Miscellaneous & $22.6 \pm 7.5^{*}$ & $16.8 \pm 7^{*}$ & $8.5 \pm 3.2^{*}$ \\
\hline
\end{tabular}

Values are presented as mean \pm SD. ADA: adenosine deaminase; ADA-2: adenosine deaminase-2. *: $\mathrm{p}<0.001$ with respect to the tuberculosis pleurisy group; $* *: \mathrm{p}<0.05$ with respect to the tuberculous pleurisy group.

otherwise), except for total ADA and 2'-deoxyadenosine deaminase activities with respect to the empyema group.

Figure 1 shows the total pleural ADA activities of each group of patients. All the tuberculous effusions had total ADA activities of $47 \mathrm{U} \cdot \mathrm{L}^{-1}$ or more, whereas 77 of the 89 patients with neoplastic disease, 51 of the 55 cases of parapneumonic effusion, 5 of the 14 empyema cases and all transudates and miscellaneous cases had total ADA activities below this level.

Figure 2 shows the pleural ADA-2 activities of each group of patients studied. All the tuberculous effusions had pleural ADA-2 activities of $40 \mathrm{U} \cdot \mathrm{L}^{-1}$ or more, whereas in 81 of the 89 neoplastic cases, 11 of the 14 empyema cases and all transudates and parapneumonic and miscellaneous cases pleural ADA-2 activity was below this level.

Figure 3 shows the pleural 2'-deoxyadenosine deaminase activities of each group of patients studied. For a threshold of $22 \mathrm{U} \cdot \mathrm{L}^{-1}, 72$ of the 76 tuberculous effusions were positive, whilst 81 of the 89 cases of neoplastic disease, 53 of the 55 parapneumonic effusions, 3 of the 14 empyema cases and all transudates and miscellaneous cases were negative.

The sensitivities of total ADA activity, ADA-2 and 2'deoxyadenosine deaminase for tuberculosis were 100, 100 and $95 \%$, respectively; their specificities 91, 96 and 92\%; and their efficiencies 93, 97 and 93\%. The 4\% differences between the efficiency of ADA-2 and those of 2'-deoxyadenosine deaminase and total ADA activity were not statistically significant.

One hundred and one effusions (all 76 tuberculous, 12 neoplastic, 4 parapneumonic and 9 empyematous exudates) had total pleural ADA activities $>47 \mathrm{U} \cdot \mathrm{L}^{-1}$; of these, 8 neoplastic, 1 parapneumonic and all the tuberculous exudates had a 2'-deoxyadenosine deaminase/ADA activity ratio $<0.49$ (sensitivity 100 , specificity 64 and efficiency $91 \%$ for tuberculosis among high-ADA effusions) (fig. 4).

The criterion of simultaneously having total ADA activity $>47 \mathrm{U} \cdot \mathrm{L}^{-1}$, ADA-2 $>40 \mathrm{U} \cdot \mathrm{L}^{-1}$ and a 2 '-deoxyadenosine deaminase/ADA activity ratio $<0.49$ was satisfied by all the tuberculous effusions but only eight others (all neoplastic) (sensitivity $100 \%$, specificity $97 \%$, efficiency 98\%).

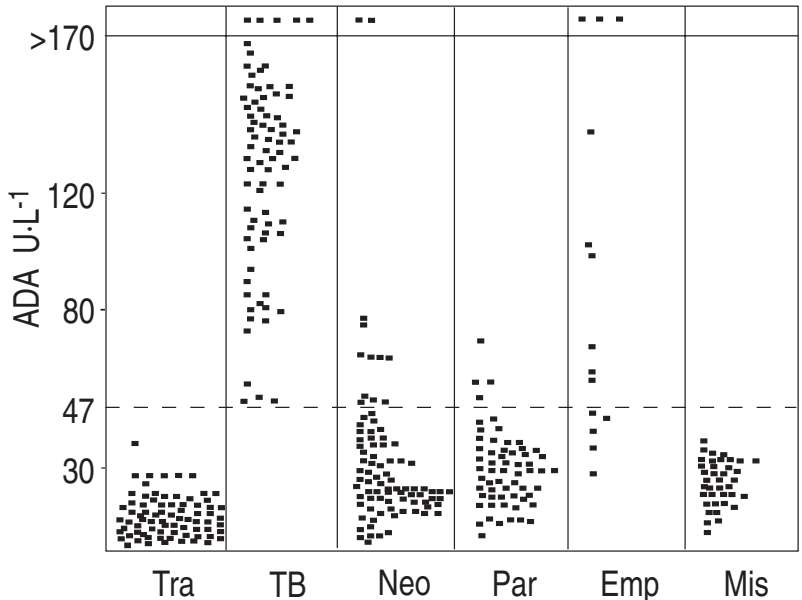

Fig. 1. - Total adenosine deaminase activity (ADA) in pleura fluid. Tra: transudates; TB: tuberculous; Neo: neoplastics; Par: parapneumonics; Emp: empyema; Mis: miscellaneous.

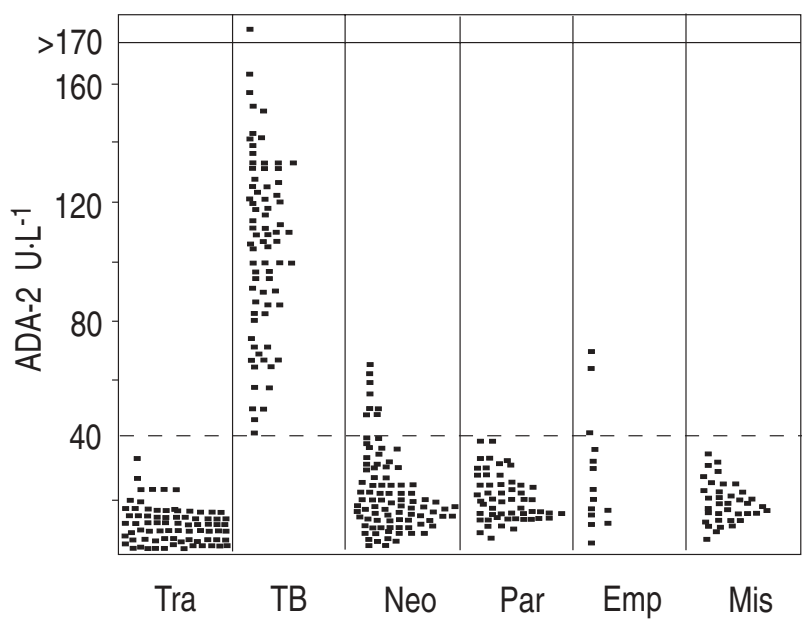

Fig. 2. - Adenosine deaminase-2 activity (ADA-2) in pleural fluid. Tra: transudates; TB: tuberculous; Neo: neoplastics; Par: parapneumonics; Emp: empyema; Mis: miscellaneous.

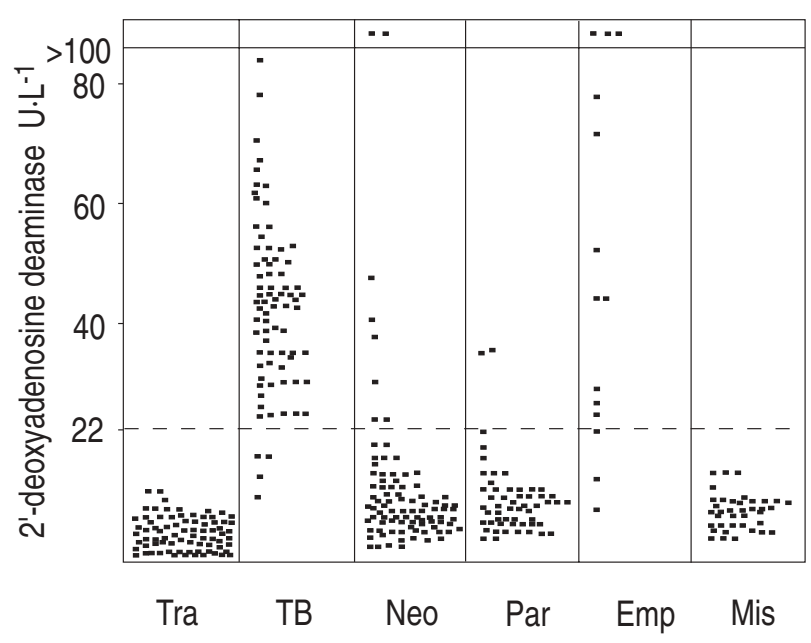

Fig. 3. - 2'-deoxyadenosine deaminase activity in pleural fluid. Tra: transudates; TB: tuberculous; Neo: neoplastics; par: parapneumonics; Emp: empyema; Mis: miscellaneous. 


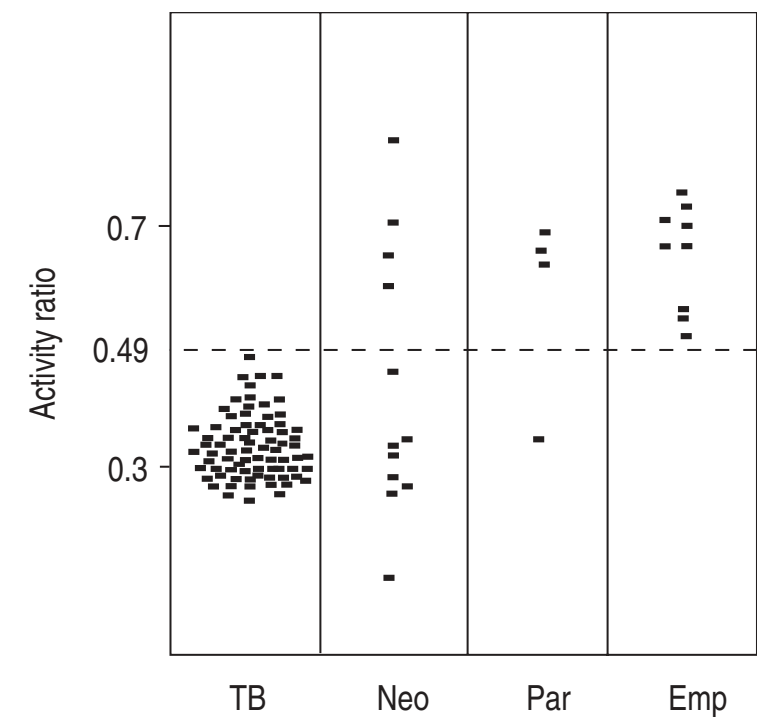

Fig. 4. - 2'-deoxyadenosine deaminase/ADA activity ratios in pleural fluid with total ADA activity greater than the established cut-off (47 U.L $\left.{ }^{-1}\right)$. TB: tuberculous; Neo: neoplastics; Par: parapneumonics; Emp: empyema.

\section{Discussion}

The above results confirm that the high total ADA activities in tuberculous pleural effusions are due largely to high ADA-2 activity, and hence to stimulation of the only known source of ADA-2, monocytes and macrophages.

The immunological reactions set off in the course of a tuberculous pleural effusion are complex and imperfectly understood. In particular, the cause of increased ADA activity in tuberculous pleural effusions is still uncertain. The suggestion that it is related to increased lymphocyte counts in these effusions appears to have been ruled out by a reported lack of correlation between ADA activity and either total lymphocyte count, T-cell, CD4 and CD 8 counts and percentages, or the CD4/CD8 ratio $[2,16,17]$. OCAÑA and co-workers [17] concluded that increased ADA activity might be due to the T-cell population of tuberculous pleural effusions being more immature and reactive than those of other effusions on account of the cellular immune response to mycobacterial antigens. However, the finding that increased ADA activity in tuberculous pleurisy is due largely to increased activity of the ADA isoenzyme ADA-2, together with the fact that the only cells in which ADA-2 has been found are monocytes/macrophages, led GAKIS and coworkers [9] to attribute increased ADA activity in tuberculous pleural effusions to the stimulation of monocytes/ macrophages by live phagocytosed micro-organisms. The unique origin of ADA-2 has recently been corroborated by UNGERER et al. [18], whilst the results now reported confirm that the increased ADA activity in tuberculous pleural effusions results largely from increased ADA-2 activity. We therefore believe that GAKIS and co-workers [9] are right in pointing to monocytes/macrophages as the origin of the increased ADA activity found in tuberculous pleural effusions.

In our study, all the tuberculous effusions had total ADA activities above $47 \mathrm{U} \cdot \mathrm{L}^{-1}$, a threshold also exceeded by 9 empyemas, 12 neoplastic exudates and 4 parapneumonic exudates. ADA-2 was a slightly more efficient indicator of tuberculous pleurisy, although the difference was not statistically significant: all the tuberculous effusions again had activities above the cut-off level $\left(40 \mathrm{U} \cdot \mathrm{L}^{-1}\right)$, and the only other effusions exceeding this level were 8 of the 12 neoplastic exudates with high total ADA activity and 3 of the 9 empyemas with high total ADA activity; the four parapneumonic exudates with high total ADA activity all had low ADA-2 activity, and no effusion with total ADA activity below $47 \mathrm{U} \cdot \mathrm{L}^{-1}$ had ADA-2 activity above $40 \mathrm{U} \cdot \mathrm{L}^{-1}$.

The high total ADA activity in 9 of the 14 empyemas was undoubtedly due largely to the large numbers of polymorphonuclear cells in these effusions. The facts that 6 of these 9 high-ADA empyemas had low ADA-2 activities, and that the mean ADA-2 activity of the nine differed significantly from that of the tuberculous group, are therefore in keeping with the ADA of polymorphonuclear cells being ADA-1. The high ADA-2 activities in the other three high-ADA empyemas merely reflect their exceptionally high total ADA activities (>170 U.L $\mathrm{L}^{-1}$ in all three cases); all nine high-ADA empyemas had a 2'-deoxyadenosine deaminase/ADA activity ratio greater than 0.49 .

The eight neoplastic exudates with ADA-2 and total ADA activities above cut-off (two associated with lymphomas, one with chronic lymphatic leukaemia, two with metastasized carcinoma of the breast, one with bronchogenic carcinoma, one with thyroid carcinoma and one with metastasis of unidentified primary origin) all had 2'-deoxyadenosine deaminase/ADA activity ratios below 0.49 . Hence ADA-2 activity, total ADA activity and the 2'-deoxyadenosine deaminase/ADA activity ratio failed to achieve $100 \%$ joint specificity as indicators of tuberculous pleurisy.

We do not know for sure what the cause of the high ADA-2 activity in eight neoplastic effusions was. However, since total ADA activity in serum is high in certain neoplastic diseases [19-21], and since most serum ADA activity is due to ADA-2 even in normal persons [18], it seems possible that the increase in capillary permeability known to occur in neoplastic pleurisy may have facilitated the passage of ADA-2 from the blood to the pleural cavity. This suggestion is supported by the fact that, of the eight patients with high-ADA, high-ADA-2 effusions, the six whose total serum ADA activity was also measured had high levels of this latter parameter (62.9 $\pm 46 \mathrm{U} \cdot \mathrm{L}^{-1}$, range $24-129 \mathrm{U} \cdot \mathrm{L}^{-1}$; normal range $14-21$ U.L $\mathrm{L}^{-1}$ ). Future studies may clarify the cause or causes of high ADA-2 and total ADA activities in neoplastic effusions.

Determination of pleural fluid 2'-deoxyadenosine deaminase activity, though a necessary step in the estimation of ADA-2 activity [15], does not seem to be of clinical utility per se, even though its diagnostic efficiency in this study was as high as that of total ADA activity and almost as high as that of ADA-2 activity. The diagnostic efficiency of the 2'-deoxyadenosine deaminase/ADA activity ratio was poor: although this ratio was $<0.49$ in all the tuberculous effusions (sensitivity $100 \%$ ), one parapneumonic and eight neoplastic effusions also had 2'deoxyadenosine deaminase/ADA activity ratios below this threshold (specificity 64\%).

In conclusion, we believe that high ADA activity in tuberculous effusions is due mainly to an increase in 
ADA-2 activity, and is therefore probably produced by monocytes/macrophages. In this study, ADA-2 activity was a more efficient marker of tuberculous effusions than was total ADA activity, but the difference was not statistically significant and the extra labour involved in the estimation of ADA-2 activity would not seem to be justifiable as routine clinical practice. A 2'-deoxyadenosine deaminase/ADA activity ratio $\geq 0.49$ appears to rule out tuberculosis, but a value below this threshold is only a very poorly specific positive indication of tuberculosis. The conjunction of the ADA-2, total ADA and 2'deoxyadenosine deaminase/ADA activity ratio criteria for tuberculosis (i.e. the requirement that ADA-2 activity be $>40 \mathrm{U} \cdot \mathrm{L}^{-1}$, total ADA activity $>47 \mathrm{U} \cdot \mathrm{L}^{-1}$ and the 2'-deoxyadenosine deaminase/ADA activity ratio $<0.49$ ), though fulfilled by all the tuberculous effusions, still resulted in false positive diagnosis of eight neoplastic effusions as tuberculosis cases. Future research should, therefore, investigate the origin of high ADA-2 and total ADA activity in neoplastic effusions.

\section{References}

1. Piras MA, Gakis C, Budroni M, Andreoni G. Adenosine deaminase activity in pleural effusions: an aid to differential diagnosis. $B r$ Med $J$ 1978; 2: 1751-1752.

2. Ocaña I, Martínez Vázquez JM, Segura RM, Fernández de Sevilla T, Capdevila JA. Adenosine deaminase in pleural fluids: test for diagnosis of tuberculous pleural effusion. Chest 1983; 84: 51-53.

3. Pettersson T, Ojala K, Weber TH. Adenosine deaminase in the diagnosis of pleural effusion. Acta Med Scand 1984; 215: 299-304.

4. Fontán J, Verea H, Pérez J, Domínguez L, Martín MT, Montero MC. Diagnostic value of simultaneous determination of pleural adenosine deaminase and pleural lysozyme/serum lysozyme ratio in pleural effusions. Chest 1988; 93: 303-307.

5. Valdés L, San José E, Alvarez D, et al. Diagnosis of tuberculous pleurisy using the biologic parameters adenosine deaminase, lysozyme and interferon-gamma. Chest 1993; 103: 458-465.

6. Ena J, Valls V, Pérez de Oteyza C, Enríquez de Salamanca R. Utilidad y limitaciones de la adenosina desaminasa en el diagnóstico de la pleuresía tuberculosa: estudio metaanalítico. Med Clin (Barc) 1990; 95: 333-335.
7. Hirschhorn R, Ratech H. Isoenzymes of adenosine deaminase. In: Ratazzi MC, Scandalia JG, Whitt GS, eds. Isoenzymes: Current Topics in Biological and Medical Research. New York, Alan R. Liss, 1980; pp. 132-157.

8. Zuckerman SH, Olson JM, Douglas SD. Adenosine deaminase activity during in vitro culture of human peripheral blood monocytes and pulmonary alveolar macrophages. Exp Cell Res 1980; 129: 281-287.

9. Gakis C, Calia G, Naitana A, Pirino D, Serru G. Serum adenosine deaminase activity in HIV positive subjects: a hypothesis on the significance of ADA-2. Panminerva Med 1989; 31: 107-113.

10. Gakis C, Calia GM, Naitana AGV, Ortu AR, Contu A. Serum and pleural adenosine deaminase activity: correct interpretation of the findings. Chest 1991; 99: 1555-1556.

11. Sahn SA. State of the art: the pleura. Am Rev Respir Dis 1988; 138: 184-234.

12. Cope C. New pleural biopsy needle. J Am Med Assoc 1958; 167: 1107-1108.

13. Abrams LD. New inventions: a pleural biopsy punch. Lancet 1958; 1: 30-31.

14. Giusti G. Adenosine deaminase. In: Bergmeyer HU, ed. Methods of Enzymatic Analysis. New York, Academic Press, 1974; pp. 1092-1099.

15. Giusti G, Gakis C. Temperature conversion factor, activation energy, relative substrate specificity and optimum $\mathrm{pH}$ of adenosine deaminase from human serum and tissue. Enzyme 1971; 12: 417-425.

16. Ocaña I, Martínez-Vázquez JM, Ribera E, Segura R, Pascual C. Adenosine deaminase activity in the diagnosis of lymphocytic pleural effusions of tuberculous, neoplastic and lymphomatous origin. Tubercle 1986; 67: 141-145.

17. Ocaña I, Ribera E, Bejarano E, Fernandez de Sevilla T, Español T, Martínez-Vázquez JM. Subpoblaciones linfocitarias en los derrames pleurales: relación con la actividad de ADA. Ann Med Intern (Madrid) 1988; 6: 274-278.

18. Ungerer JPJ, Oosthuizen HM, Bissbort SH, Vermaak WJH. Serum adenosine deaminase: isoenzymes and diagnostic application. Clin Chem 1992; 38: 1322-326.

19. Koehler LH, Benz EJ. Serum adenosine deaminase: methodology and clinical applications. Clin Chem 1962; 8: $133-140$.

20. Giusti G, Galanti B. Colorimetric method. In: Bergmeyer HU, ed. Methods of Enzymatic Analysis, 3rd edn. Weinheim, Verlag Chemie, 1984: 315-323.

21. Goldberg DM, Fletcher MJ, Watts C. Serum adenosine deaminase activity in hepatic disease: a comparative enzymological evaluation. Clin Chim Acta 1966; 14: $720-728$. 